\title{
Generalized Characteristic Polynomials of Join Graphs and Their Applications
}

\author{
Pengli Lu, Ke Gao, and Yang Yang \\ School of Computer and Communication, Lanzhou University of Technology, Lanzhou, Gansu 730050, China \\ Correspondence should be addressed to Pengli Lu; lupengli88@163.com
}

Received 11 November 2016; Revised 18 January 2017; Accepted 26 January 2017; Published 2 March 2017

Academic Editor: Francisco R. Villatoro

Copyright (C) 2017 Pengli Lu et al. This is an open access article distributed under the Creative Commons Attribution License, which permits unrestricted use, distribution, and reproduction in any medium, provided the original work is properly cited.

\begin{abstract}
The Kirchhoff index of $G$ is the sum of resistance distances between all pairs of vertices of $G$ in electrical networks. LEL $(G)$ is the Laplacian-Energy-Like Invariant of $G$ in chemistry. In this paper, we define two classes of join graphs: the subdivision-vertexvertex join $G_{1} \odot G_{2}$ and the subdivision-edge-edge join $G_{1} \odot G_{2}$. We determine the generalized characteristic polynomial of them. We deduce the adjacency (Laplacian and signless Laplacian, resp.) characteristic polynomials of $G_{1} \odot G_{2}$ and $G_{1} \odot G_{2}$ when $G_{1}$ is $r_{1}$-regular graph and $G_{2}$ is $r_{2}$-regular graph. As applications, the Laplacian spectra enable us to get the formulas of the number of spanning trees, Kirchhoff index, and LEL of $G_{1} \odot G_{2}$ and $G_{1} \odot G_{2}$ in terms of the Laplacian spectra of $G_{1}$ and $G_{2}$.
\end{abstract}

\section{Introduction}

Let $G=(V(G), E(G))$ be a simple graph on $n$ vertices and $m$ edges. Let $d_{i}=d_{G}\left(v_{i}\right)$ be the degree of vertex $v_{i}$ in $G$ and $D(G)$ be the diagonal matrix with diagonal entries $d_{1}, d_{2}, \ldots, d_{n}$. Let $A(G)$ denote the adjacency matrix of a graph $G$. The Laplacian matrix and the signless Laplacian matrix of $G$ are defined as $L(G)=D(G)-A(G)$ and $Q(G)=$ $D(G)+A(G)$, respectively. Let $\psi(A(G) ; x)=\operatorname{det}\left(x I_{n}-\right.$ $A(G))$, or simply $\psi(A(G))(\psi(L(G))$ and $\psi(Q(G))$, resp.), be the adjacency (Laplacian and signless Laplacian, resp.) characteristic polynomial of $G$ and its roots be the adjacency (Laplacian and signless Laplacian, resp.) eigenvalues of $G$, denoted by $\lambda_{1}(G) \geq \lambda_{2}(G) \geq \cdots \geq \lambda_{n}(G)\left(0=\mu_{1}(G) \leq\right.$ $\mu_{2}(G) \leq \cdots \leq \mu_{n}(G)$ and $\nu_{1}(G) \leq \nu_{2}(G) \leq \cdots \leq \nu_{n}(G)$, resp.). The line graph of $G$ is denoted by $\ell(G)$.

The generalized characteristic polynomial of $G$, introduced by Cvetkovi et al. [1], is defined to be $\phi_{G}(x, t)=\operatorname{det}\left(x I_{n}-\right.$ $(A(G)-t D(G)))$. The generalized characteristic polynomial covers the cases of usual characteristic polynomial $A(G)$ and Laplacian $L(G)$ and signless Laplacian $Q(G)$ polynomials of graph $G$, due to variation of the parameter $t$ and, whenever necessary, replacing $x$ by $-x$. We can get that the characteristic polynomials of $A(G), L(G)$, and $Q(G)$ are equal to $\phi_{G}(x, 0)$, $(-1)^{|V(G)|} \phi_{G}(-x, 1)$, and $\phi_{G}(x,-1)$.
Let $G$ be a connected graph. For two vertices $u$ and $v$ of $G$, the resistance distance between $u$ and $v$ is defined to be the effective resistance between them when unit resistors are placed on every edge of $G$. It is a distance function on graphs introduced by Klein and Randić [2]. The Kirchhoff index of $G$, denoted by $\operatorname{Kf}(G)$, is the sum of resistance distances between all pairs of vertices of $G$. For a connected graph $G$ of order $n$ [3], $\operatorname{Kf}(G)=n \sum_{i=2}^{n}\left(1 / \mu_{i}\right)$. Recently, many results on Kirchhoff index were obtained [2, 4-8]. LaplacianEnergy-Like Invariant LEL $(G)=\sum_{i=2}^{n} \sqrt{\mu_{i}}$ was named in [9]. The motivation for introducing LEL was in its analogy to the earlier studied graph energy and Laplacian energy [10]. Although Kirchhoff index and LEL both depend on Laplacian eigenvalues, their comparative study started only quite recently [11, 12].

Graph operations, such as the disjoint union, the join, the corona, the edge corona, and the neighborhood corona [13-17], are techniques to construct new classes of graphs from old ones. In [18], a real molecular graph of ferrocene is a join graph obtained from graphs $G_{1}=K_{1}$ and $G_{2}=2 C_{5}$, where $2 C_{5}$ is a disjoint union of two pentagons. In $[4-6,8,14]$ the resistance distance and Kirchhoff index of artificial graphs are computed. Although most of the constructed graphs in the literature are contrived, they may be of use for chemical and physical applications. 


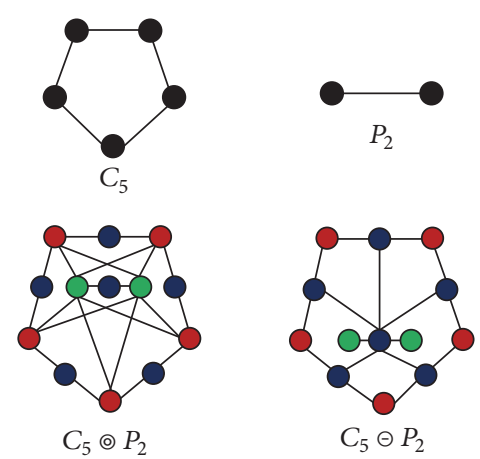

FIgURE 1: An example of subdivision-vertex-vertex join and subdivision-edge-edge join.

Motivated by the work above, we define two new graph operations based on subdivision graphs as follows.

The subdivision graph $\mathcal{S}(G)$ of a graph $G$ is the graph obtained by inserting a new vertex into every edge of $G$ [19]. We denote the set of such new vertices by $I(G)$. In $[15,20]$, some new graph operations based on subdivision graphs were defined and the $A^{-}, L^{-}$, and $Q$-spectrum were computed in terms of those of the two graphs.

Let $G_{1}$ and $G_{2}$ be two vertex disjoint graphs. The subdivision-vertex-vertex join of $G_{1}$ and $G_{2}$, denoted by $G_{1} \odot G_{2}$, is the graph obtained from $\mathcal{S}\left(G_{1}\right)$ and $\mathcal{S}\left(G_{2}\right)$ by joining every vertex in $V\left(G_{1}\right)$ to every vertex in $V\left(G_{2}\right)$. The subdivision-edge-edge join of $G_{1}$ and $G_{2}$, denoted by $G_{1} \odot G_{2}$, is the graph obtained from $\mathcal{S}\left(G_{1}\right)$ and $\mathcal{S}\left(G_{2}\right)$ by joining every vertex of $I\left(G_{1}\right)$ to every vertex in $I\left(G_{2}\right)$.

Note that if $G_{1}$ is a graph on $n_{1}$ vertices and $m_{1}$ edges and $G_{2}$ is a graph on $n_{2}$ vertices and $m_{2}$ edges, then $G_{1} \odot G_{2}$ has $n_{1}+m_{1}+n_{2}+m_{2}$ vertices and $2 m_{1}+2 m_{2}+n_{1} n_{2}$ edges and $G_{1} \odot G_{2}$ has $n_{1}+m_{1}+n_{2}+m_{2}$ vertices and $2 m_{1}+2 m_{2}+m_{1} m_{2}$ edges.

Let $C_{n}$ denote a cycle of order $n$ and $P_{m}$ denote a path of order $m$. Figure 1 depicts the subdivision-vertex-vertex join $C_{5} \odot P_{2}$ and subdivision-edge-edge join $C_{5} \odot P_{2}$, respectively.

The paper is organized as follows. In Section 2, some useful lemmas are provided. In Section 3, we compute the generalized characteristic polynomial of the subdivision-vertexvertex join and obtain the $A$-, $L$-, and $Q$-spectrum in terms of the corresponding spectrum of $G_{1}$ and $G_{2}$ when $G_{1}$ and $G_{2}$ are regular graphs. In Section 4 , we compute the generalized characteristic polynomial of the subdivision-edge-edge join and obtain the $A$-, $L$-, and $Q$-spectrum in terms of the corresponding spectrum of $G_{1}$ and $G_{2}$ when $G_{1}$ and $G_{2}$ are regular graphs. In Section 5, as the applications, the number of spanning trees, Kirchhoff index, and LEL of the subdivisionvertex-vertex join and the subdivision-edge-edge join graphs are computed. In Section 6, we conclude the paper.

\section{Preliminaries}

The $M$-coronal $\Gamma_{M}(x)$ of an $n \times n$ matrix $M$ is defined [16] to be the sum of entries of the matrix $\left(x I_{n}-M\right)^{-1}$; that is

$$
\Gamma_{M}(x)=\mathbf{1}_{n}^{T}\left(x I_{n}-M\right)^{-1} \mathbf{1}_{n},
$$

where $\mathbf{1}_{n}$ denotes the column vector of size $n$ when all the entries are equal to one.

Lemma 1 (see [21]). Let $A$ be an $n \times n$ real matrix and let $J_{s \times t}$ denote the $s \times t$ matrix with all entries equal to one. Then

$$
\operatorname{det}\left(A+\alpha J_{n \times n}\right)=\operatorname{det}(A)+\alpha \mathbf{1}_{n}^{T} \operatorname{adj}(A) \mathbf{1}_{n},
$$

where $\alpha$ is a real number and $\operatorname{adj}(A)$ is the adjugate matrix of A. Moreover,

$$
\operatorname{det}\left(x I_{n}-A-\alpha J_{n \times n}\right)=\left(1-\alpha \Gamma_{A}(x)\right) \operatorname{det}\left(x I_{n}-A\right) .
$$

Lemma 2 (see [13]). If $M$ is an $n \times n$ matrix when each row sum is equal to a constant $t$; then,

$$
\Gamma_{M}(x)=\frac{n}{x-t}
$$

Lemma 3 (see [22]). Let $M_{1}, M_{2}, M_{3}$, and $M_{4}$ be, respectively, $p \times p, p \times q, q \times p$, and $q \times q$ matrices with $M_{1}$ and $M_{4}$ invertible. Then

$$
\begin{aligned}
\operatorname{det}\left(\begin{array}{ll}
M_{1} & M_{2} \\
M_{3} & M_{4}
\end{array}\right) & =\operatorname{det}\left(M_{4}\right) \cdot \operatorname{det}\left(M_{1}-M_{2} M_{4}^{-1} M_{3}\right) \\
& =\operatorname{det}\left(M_{1}\right) \cdot \operatorname{det}\left(M_{4}-M_{3} M_{1}^{-1} M_{2}\right)
\end{aligned}
$$

where $M_{1}-M_{2} M_{4}^{-1} M_{3}$ and $M_{4}-M_{3} M_{1}^{-1} M_{2}$ are called the Schur complements of $M_{4}$ and $M_{1}$, respectively.

Lemma 4 (see [19]). If $G$ is a regular graph of degree $r$, with $n$ vertices and $m$ edges; then

$$
\psi(A(\ell(G)), x)=(x+2)^{m-n} \psi(A(G), x-r+2) .
$$

If $A=\left[a_{i j}\right]$ is an $m \times n$ matrix and $B$ is an $r \times s$ matrix, then the Kronecker product $A \otimes B$ is defined as the $m r \times n s$ matrix with the block form

$$
\left[\begin{array}{ccc}
a_{11} B & \ldots & a_{1 n} B \\
\vdots & \ddots & \vdots \\
a_{m 1} B & \ldots & a_{m n} B
\end{array}\right] .
$$

This is an associative operation with the property that $(A \otimes B)^{T}=A^{T} \otimes B^{T}$ and $(A \otimes B)(C \otimes D)=A C \otimes B D$ whenever the products $A C$ and $B D$ exist. The latter implies $(A \otimes B)^{-1}=A^{-1} \otimes B^{-1}$ for nonsingular matrices $A$ and $B$. Moreover, if $A$ and $B$ are $n \times n$ and $p \times p$ matrices, then $\operatorname{det}(A \otimes B)=(\operatorname{det} A)^{p}(\operatorname{det} B)^{n}$. The reader is referred to [23] for other properties of the Kronecker product not mentioned here.

\section{Spectrum of Subdivision-Vertex-Vertex Join}

Let $G_{1}$ be an $r_{1}$-regular graph on $n_{1}$ vertices and $m_{1}$ edges and $G_{2}$ an $r_{2}$-regular graph on $n_{2}$ vertices and $m_{2}$ edges, respectively. We first label the vertices of $G_{1} \odot G_{2}$ as follows. 
Let $V\left(G_{1}\right)=\left\{v_{1}, v_{2}, \ldots, v_{n_{1}}\right\}, I\left(G_{1}\right)=\left\{e_{1}, e_{2}, \ldots, e_{m_{1}}\right\}$, $V\left(G_{2}\right)=\left\{u_{1}, u_{2}, \ldots, u_{n_{2}}\right\}$, and $I\left(G_{2}\right)=\left\{e_{1}, e_{2}, \ldots, e_{m_{2}}\right\}$. The adjacency matrix of $G_{1} \odot G_{2}$ can be written as follows:

$$
A_{G_{1} \odot G_{2}}=\left(\begin{array}{cccc}
0_{m_{1} \times m_{1}} & R_{1}^{T} & 0_{m_{1} \times n_{2}} & 0_{m_{1} \times m_{2}} \\
R_{1} & 0_{n_{1} \times n_{1}} & \mathbf{1}_{n_{1} \times n_{2}} & 0_{n_{1} \times m_{2}} \\
0_{n_{2} \times m_{1}} & \mathbf{1}_{n_{2} \times n_{1}} & 0_{n_{2} \times n_{2}} & R_{2} \\
0_{m_{2} \times m_{1}} & 0_{m_{2} \times n_{1}} & R_{2}^{T} & 0_{m_{2} \times m_{2}}
\end{array}\right),
$$

where $0_{s \times t}$ denotes the $s \times t$ matrix with all entries equal to zero, $R$ is the incidence matrix of $G, I_{n}$ is the identity matrix of order $n$, and $\mathbf{1}_{n}$ is the column vector with all entries equal to 1 . It is clear that the degrees of the vertices of $G_{1} \odot G_{2}$ are $d_{G_{1} \odot G_{2}}\left(v_{i}\right)=r_{1}+n_{2}$ for $i=1,2, \ldots, n_{1}, d_{G_{1} \odot G_{2}}\left(e_{i}\right)=2$ for $i=1,2, \ldots, m_{1}, d_{G_{1} \odot G_{2}}\left(u_{i}\right)=r_{2}+n_{1}$ for $i=1,2, \ldots, n_{2}$, and $d_{G_{1} \odot G_{2}}\left(e_{i}\right)=2$ for $i=1,2, \ldots, m_{2}$. Then the degree matrix of subdivision-vertex-vertex join can be written as follows:

$$
\begin{aligned}
& D_{G_{1} \odot G_{2}} \\
& \quad=\left(\begin{array}{cccc}
2 I_{m_{1}} & 0_{m_{1} \times n_{1}} & 0_{m_{1} \times n_{2}} & 0_{m_{1} \times m_{2}} \\
0_{n_{1} \times m_{1}} & \left(r_{1}+n_{2}\right) I_{n_{1}} & 0_{n_{1} \times n_{2}} & 0_{n_{1} \times m_{2}} \\
0_{n_{2} \times m_{1}} & 0_{n_{2} \times n_{1}} & \left(r_{2}+n_{1}\right) I_{n_{2}} & 0_{n_{2} \times m_{2}} \\
0_{m_{2} \times m_{1}} & 0_{m_{2} \times n_{1}} & 0_{m_{2} \times n_{2}} & 2 I_{m_{2}}
\end{array}\right) .
\end{aligned}
$$

\subsection{Generalized Characteristic Polynomial of $G_{1} \odot G_{2}$}

Theorem 5. Let $G_{1}$ be an $r_{1}$-regular graph on $n_{1}$ vertices and $m_{1}$ edges and $G_{2}$ be an $r_{2}$-regular graph on $n_{2}$ vertices and $m_{2}$ edges. Then the generalized characteristic polynomial of subdivision-vertex-vertex join of $G_{1}$ and $G_{2}$ is

$$
\begin{aligned}
\phi_{G_{1} \odot G_{2}}(x, t)= & (x+2 t)^{m_{1}+m_{2}-n_{1}-n_{2}} \prod_{i=1}^{n_{1}}\left(x^{2}+\left(2 t+r_{1} t+n_{2} t\right) x+2 r_{1} t^{2}+2 n_{2} t^{2}-\lambda_{i}\left(G_{1}\right)-r_{1}\right) \\
& \cdot \prod_{i=1}^{n_{2}}\left(x^{2}+\left(2 t+r_{2} t+n_{1} t\right) x+2 r_{2} t^{2}+2 n_{1} t^{2}-\lambda_{i}\left(G_{2}\right)-r_{2}\right) \\
& \cdot\left(1-\frac{n_{1} n_{2}(x+2 t)^{2}}{\left(\left(x+r_{1} t+n_{2} t\right)(x+2 t)-2 r_{1}\right)\left(\left(x+r_{2} t+n_{1} t\right)(x+2 t)-2 r_{2}\right)}\right) .
\end{aligned}
$$

Proof. Let $R_{1}$ be the incidence matrix of $G_{1}$. Then, with respect to the adjacent matrix and degree matrix of $G_{1} \odot G_{2}$, the generalized matrix of $G_{1} \odot G_{2}$ is given by
Thus the generalized characteristic polynomial of $G_{1} \odot G_{2}$ is

$$
\begin{aligned}
& A_{G_{1} \odot G_{2}}-t D_{G_{1} \odot G_{2}} \\
& =\left(\begin{array}{cccc}
-2 t I_{m_{1}} & R_{1}^{T} & 0_{m_{1} \times n_{2}} & 0_{m_{1} \times m_{2}} \\
R_{1} & -\left(r_{1}+n_{2}\right) t I_{n_{1}} & \mathbf{1}_{n_{1} \times n_{2}} & 0_{n_{1} \times m_{2}} \\
0_{n_{2} \times m_{1}} & \mathbf{1}_{n_{2} \times n_{1}} & -\left(r_{2}+n_{1}\right) t I_{n_{2}} & R_{2} \\
0_{m_{2} \times m_{1}} & 0_{m_{2} \times n_{1}} & R_{2}^{T} & -2 t I_{m_{2}}
\end{array}\right) .
\end{aligned}
$$

$$
\begin{aligned}
\phi_{G_{1} \odot G_{2}}(x, t) & =\operatorname{det}\left(\begin{array}{cccc}
(x+2 t) I_{m_{1}} & -R_{1}^{T} & 0_{m_{1} \times n_{2}} & 0_{m_{1} \times m_{2}} \\
-R_{1} & \left(x+r_{1} t+n_{2} t\right) I_{n_{1}} & -\mathbf{1}_{n_{1} \times n_{2}} & 0_{n_{1} \times m_{2}} \\
0_{n_{2} \times m_{1}} & -\mathbf{1}_{n_{2} \times n_{1}} & \left(x+r_{2} t+n_{1} t\right) I_{n_{2}} & -R_{2} \\
0_{m_{2} \times m_{1}} & 0_{m_{2} \times n_{1}} & -R_{2}^{T} & (x+2 t) I_{m_{2}}
\end{array}\right)=\operatorname{det}\left((x+2 t) I_{m_{1}}\right) \operatorname{det}(S) \\
& =(x+2 t)^{m_{1}} \operatorname{det}(S),
\end{aligned}
$$


where

$$
\begin{aligned}
S & =\left(\begin{array}{ccc}
\left(x+r_{1} t+n_{2} t\right) I_{n_{1}} & -\mathbf{1}_{n_{1} \times n_{2}} & 0_{n_{1} \times m_{2}} \\
-\mathbf{1}_{n_{2} \times n_{1}} & \left(x+r_{2} t+n_{1} t\right) I_{n_{2}} & -R_{2} \\
0_{m_{2} \times n_{1}} & -R_{2}^{T} & (x+2 t) I_{m_{2}}
\end{array}\right)-\left(\begin{array}{c}
-R_{1} \\
0_{n_{2} \times n_{1}} \\
0_{m_{2} \times n_{1}}
\end{array}\right) \cdot\left((x+2 t) I_{m_{1}}\right)^{-1}\left(\begin{array}{ll}
-R_{1}^{T} & 0_{m_{1} \times n_{1}} \\
\left.0_{m_{1} \times m_{2}}\right)
\end{array}\right. \\
& =\left(\begin{array}{ccc}
\left(x+r_{1} t+n_{2} t\right) I_{n_{1}}-\frac{R_{1} R_{1}^{T}}{x+2 t} & -\mathbf{1}_{n_{1} \times n_{2}} & 0_{n_{1} \times m_{2}} \\
-\mathbf{1}_{n_{2} \times n_{1}} & \left(x+r_{2} t+n_{1} t\right) I_{n_{2}} & -R_{2} \\
0_{m_{2} \times n_{1}} & -R_{2}^{T} & (x+2 t) I_{m_{2}}
\end{array}\right)
\end{aligned}
$$

is the Schur complement of $(x+2 t) I_{m_{1}}$. So, $\operatorname{det}(S)=(x+$ $2 t)^{m_{2}} \operatorname{det}\left(S_{1}\right)$, where

$S_{1}$

$$
\begin{aligned}
& =\left(\begin{array}{cc}
\left(x+r_{1} t+n_{2} t\right) I_{n_{1}}-\frac{R_{1} R_{1}^{T}}{x+2 t} & -\mathbf{1}_{n_{1} \times n_{2}} \\
-\mathbf{1}_{n_{2} \times n_{1}} & \left(x+r_{2} t+n_{1} t\right) I_{n_{2}}
\end{array}\right) \\
& =\left(\begin{array}{c}
0_{n_{1} \times m_{2}} \\
-R_{2}
\end{array}\right)\left((x+2 t) I_{m_{2}}\right)^{-1}\left(\begin{array}{ll}
0_{m_{2} \times n_{1}} & \left.-R_{2}^{T}\right)
\end{array}\right. \\
& =\left(\begin{array}{cc}
\left(x+r_{1} t+n_{2} t\right) I_{n_{1}}-\frac{R_{1} R_{1}^{T}}{x+2 t} & -\mathbf{1}_{n_{1} \times n_{2}} \\
-\mathbf{1}_{n_{2} \times n_{1}} & \left(x+r_{2} t+n_{1} t\right) I_{n_{2}}-\frac{R_{2} R_{2}^{T}}{x+2 t}
\end{array}\right)
\end{aligned}
$$

is the Schur complement of $(x+2 t) I_{m_{2}}$. So,

$$
\begin{aligned}
& \operatorname{det}\left(S_{1}\right)=\operatorname{det}\left(\left(x+r_{1} t+n_{2} t\right) I_{n_{1}}-\frac{R_{1} R_{1}^{T}}{x+2 t}\right) \\
& \cdot \operatorname{det}\left(\left(x+r_{2} t+n_{1} t\right) I_{n_{2}}-\frac{R_{2} R_{2}^{T}}{x+2 t}\right. \\
& \left.-\mathbf{1}_{n_{2} \times n_{1}}\left(\left(x+r_{1} t+n_{2} t\right) I_{n_{1}}-\frac{R_{1} R_{1}^{T}}{x+2 t}\right)^{-1} \mathbf{1}_{n_{1} \times n_{2}}\right)
\end{aligned}
$$

is the Schur complement of $\left(x+r_{1} t+n_{2} t\right) I_{n_{1}}-R_{1} R_{1}^{T} /(x+2 t)$. So,

$$
\begin{aligned}
& \phi_{G_{1} \odot G_{2}}(x, t)=(x+2 t)^{m_{1}+m_{2}} \operatorname{det}\left(\left(x+r_{1} t+n_{2} t\right) I_{n_{1}}\right. \\
& \left.-\frac{R_{1} R_{1}^{T}}{x+2 t}\right) \cdot \operatorname{det}\left(\left(x+r_{2} t+n_{1} t\right) I_{n_{2}}-\frac{R_{2} R_{2}^{T}}{x+2 t}\right. \\
& \left.-\mathbf{1}_{n_{2} \times n_{1}}\left(\left(x+r_{1} t+n_{2} t\right) I_{n_{1}}-\frac{R_{1} R_{1}^{T}}{x+2 t}\right)^{-1} \mathbf{1}_{n_{1} \times n_{2}}\right) \\
& =(x+2 t)^{m_{1}+m_{2}} \operatorname{det}\left(\left(x+r_{1} t+n_{2} t\right) I_{n_{1}}\right. \\
& \left.-\frac{A_{G_{1}}+D_{G_{1}}}{x+2 t}\right) \cdot \operatorname{det}\left(\left(x+r_{2} t+n_{1} t\right) I_{n_{2}}-\frac{R_{2} R_{2}^{T}}{x+2 t}\right. \\
& \left.-\mathbf{1}_{n_{2} \times n_{1}}\left(\left(x+r_{1} t+n_{2} t\right) I_{n_{1}}-\frac{R_{1} R_{1}^{T}}{x+2 t}\right)^{-1} \mathbf{1}_{n_{1} \times n_{2}}\right) \\
& =(x+2 t)^{m_{1}+m_{2}} \prod_{i=1}^{n_{1}}\left(x+r_{1} t+n_{2} t-\frac{\lambda_{i}\left(G_{1}\right)+r_{1}}{x+2 t}\right) \\
& \cdot \operatorname{det}\left(\left(x+r_{2} t+n_{1} t\right) I_{n_{2}}-\frac{R_{2} R_{2}^{T}}{x+2 t}\right. \\
& \left.-\Gamma_{R_{1} R_{1}^{T} /(x+2 t)}\left(x+r_{1} t+n_{2} t\right) \mathbf{1}_{n_{2} \times n_{2}}\right) \text {; }
\end{aligned}
$$

by Lemma 1 ,

$$
\begin{aligned}
\phi_{G_{1} \odot G_{2}}(x, t)= & (x+2 t)^{m_{1}+m_{2}} \prod_{i=1}^{n_{1}}\left(x+r_{1} t+n_{2} t-\frac{\lambda_{i}\left(G_{1}\right)+r_{1}}{x+2 t}\right) \\
& \cdot\left(1-\Gamma_{R_{1} R_{1}^{T} /(x+2 t)}\left(x+r_{1} t+n_{2} t\right) \Gamma_{R_{2} R_{2}^{T} /(x+2 t)}\left(x+r_{2} t+n_{1} t\right)\right) \operatorname{det}\left(\left(x+r_{2} t+n_{1} t\right) I_{n_{2}}-\frac{R_{2} R_{2}^{T}}{x+2 t}\right) \\
= & \prod_{i=1}^{n_{1}}\left(x^{2}+\left(2 t+r_{1} t+n_{2} t\right) x+2 r_{1} t^{2}+2 n_{2} t^{2}-\lambda_{i}\left(G_{1}\right)-r_{1}\right)
\end{aligned}
$$




$$
\begin{aligned}
& \cdot \prod_{i=1}^{n_{2}}\left(x^{2}+\left(2 t+r_{2} t+n_{1} t\right) x+2 r_{2} t^{2}+2 n_{1} t^{2}-\lambda_{i}\left(G_{2}\right)-r_{2}\right) \\
& \cdot\left(1-\Gamma_{R_{1} R_{1}^{T} /(x+2 t)}\left(x+r_{1} t+n_{2} t\right) \Gamma_{R_{2} R_{2}^{T} /(x+2 t)}\left(x+r_{2} t+n_{1} t\right)\right)(x+2 t)^{m_{1}+m_{2}-n_{1}-n_{2}} \\
= & (x+2 t)^{m_{1}+m_{2}-n_{1}-n_{2}} \prod_{i=1}^{n_{1}}\left(x^{2}+\left(2 t+r_{1} t+n_{2} t\right) x+2 r_{1} t^{2}+2 n_{2} t^{2}-\lambda_{i}\left(G_{1}\right)-r_{1}\right) \\
& \cdot \prod_{i=1}^{n_{2}}\left(x^{2}+\left(2 t+r_{2} t+n_{1} t\right) x+2 r_{2} t^{2}+2 n_{1} t^{2}-\lambda_{i}\left(G_{2}\right)-r_{2}\right) \\
& \cdot\left(1-\frac{n_{1} n_{2}(x+2 t)^{2}}{\left(\left(x+r_{1} t+n_{2} t\right)(x+2 t)-2 r_{1}\right)\left(\left(x+r_{2} t+n_{1} t\right)(x+2 t)-2 r_{2}\right)}\right) .
\end{aligned}
$$

Theorem 6. Let $G_{1}$ be an $r_{1}$-regular graph on $n_{1}$ vertices and $m_{1}$ edges and $G_{2}$ an $r_{2}$-regular graph on $n_{2}$ vertices and $m_{2}$ edges. Then

(1) the adjacency characteristic polynomial of $G_{1} \odot G_{2}$ is

$$
\begin{aligned}
\psi & \left(A_{G_{1} \odot G_{2}} ; x\right)=x^{m_{1}+m_{2}-n_{1}-n_{2}} \prod_{i=1}^{n_{1}}\left(x^{2}-r_{1}-\lambda_{i}\left(G_{1}\right)\right) \\
& \cdot \prod_{i=1}^{n_{2}}\left(x^{2}-r_{2}-\lambda_{i}\left(G_{2}\right)\right) \\
& \cdot\left(1-\frac{n_{1} n_{2} x^{2}}{x^{4}-2\left(r_{1}+r_{2}\right) x^{2}+4 r_{1} r_{2}}\right)
\end{aligned}
$$

(2) the Laplacian characteristic polynomial of $G_{1} \odot G_{2}$ is

$$
\begin{aligned}
\psi & \left(L_{G_{1} \odot G_{2}} ; x\right) \\
& =\prod_{i=2}^{n_{1}}\left(x^{2}-\left(r_{1}+n_{2}+2\right) x+2 n_{2}+\mu_{i}\left(G_{1}\right)\right) \\
& \cdot \prod_{i=2}^{n_{2}}\left(x^{2}-\left(r_{2}+n_{1}+2\right) x+2 n_{1}+\mu_{i}\left(G_{2}\right)\right) \cdot x\left(x^{3}\right. \\
& -\left(n_{1}+n_{2}+r_{1}+r_{2}+4\right) x^{2} \\
& +\left(4 n_{1}+4 n_{2}+2 r_{1}+2 r_{2}+4+r_{1} r_{2}+r_{1} n_{1}+r_{2} n_{2}\right) \\
& \left.\cdot x-4 n_{1}-4 n_{2}-2 n_{1} r_{1}-2 n_{2} r_{2}\right)(x-2)^{m_{1}+m_{2}-n_{1}-n_{2}},
\end{aligned}
$$

(3) the signless Laplacian characteristic polynomial of $G_{1} \odot$ $G_{2}$ is

$$
\begin{aligned}
& \psi\left(Q_{G_{1} \odot G_{2}} ; x\right)=(x-2)^{m_{1}+m_{2}-n_{1}-n_{2}} \\
& \quad \cdot \prod_{i=1}^{n_{1}}\left(x^{2}-\left(r_{1}+n_{2}+2\right) x+2 n_{2}+2 r_{1}-v_{i}\left(G_{1}\right)\right) \cdot(1
\end{aligned}
$$

$$
\begin{aligned}
& \left.-\frac{n_{1} n_{2}(x-2)^{2}}{\left(x^{2}-\left(2+r_{1}+n_{2}\right) x+2 n_{2}\right)\left(x^{2}-\left(2+r_{2}+n_{1}\right) x+2 n_{1}\right)}\right) \\
& \cdot \prod_{i=1}^{n_{2}}\left(x^{2}-\left(r_{2}+n_{1}+2\right) x+2 n_{1}+2 r_{2}-v_{i}\left(G_{2}\right)\right) .
\end{aligned}
$$

\section{Spectrum of Subdivision-Edge-Edge Join}

The adjacency matrix of $G_{1} \odot G_{2}$ can be written as follows:

$$
A_{G_{1} \ominus G_{2}}=\left(\begin{array}{cccc}
0_{n_{1} \times n_{1}} & R_{1} & 0_{n_{1} \times m_{2}} & 0_{n_{1} \times n_{2}} \\
R_{1}^{T} & 0_{m_{1} \times m_{1}} & \mathbf{1}_{m_{1} \times m_{2}} & 0_{m_{1} \times n_{2}} \\
0_{m_{2} \times n_{1}} & \mathbf{1}_{m_{2} \times m_{1}} & 0_{m_{2} \times m_{2}} & R_{2}^{T} \\
0_{n_{2} \times n_{1}} & 0_{n_{2} \times m_{1}} & R_{2} & 0_{n_{2} \times n_{2}}
\end{array}\right),
$$

where $0_{s \times t}$ denotes the $s \times t$ matrix with all entries equal to zero, $R$ is the incidence matrix of $G, I_{n}$ is the identity matrix of order $n$, and $\mathbf{1}_{n}$ is the column vector with all entries equal to 1 . It is clear that the degrees of the vertices of $G_{1} \odot G_{2}$ are $d_{G_{1} \odot G_{2}}\left(v_{i}\right)=r_{1}$ for $i=1,2, \ldots, n_{1}, d_{G_{1} \odot G_{2}}\left(e_{i}\right)=m_{2}+2$ for $i=1,2, \ldots, m_{1}, d_{G_{1} \odot G_{2}}\left(u_{i}\right)=r_{2}$ for $i=1,2, \ldots, n_{2}$, and $d_{G_{1} \ominus G_{2}}\left(e_{i}\right)=m_{1}+2$ for $i=1,2, \ldots, m_{2}$. Then the degree matrix of subdivision-edge-edge join can be written as follows:

$$
\begin{aligned}
& D_{G_{1} \ominus G_{2}} \\
& =\left(\begin{array}{cccc}
r_{1} I_{n_{1}} & 0_{n_{1} \times m_{1}} & 0_{n_{1} \times m_{1}} & 0_{n_{1} \times n_{2}} \\
0_{m_{1} \times n_{1}} & \left(m_{2}+2\right) I_{m_{1}} & 0_{m_{1} \times m_{2}} & 0_{m_{1} \times n_{2}} \\
0_{m_{2} \times n_{1}} & 0_{m_{2} \times m_{1}} & \left(m_{1}+2\right) I_{m_{2}} & 0_{m_{2} \times n_{2}} \\
0_{n_{2} \times n_{1}} & 0_{n_{2} \times m_{1}} & 0_{n_{2} \times m_{2}} & r_{2} I_{n_{2}}
\end{array}\right) .
\end{aligned}
$$


4.1. Generalized Characteristic Polynomial of $G_{1} \odot G_{2}$

Theorem 7. Let $G_{1}$ be an $r_{1}$-regular graph on $n_{1}$ vertices and $m_{1}$ edges and $G_{2}$ be an $r_{2}$-regular graph on $n_{2}$ vertices and $m_{2}$ edges. Then the generalized characteristic polynomial of subdivision-edge-edge join of $G_{1}$ and $G_{2}$ is

$$
\begin{aligned}
\phi_{G_{1} \odot G_{2}}(x ; t)= & \prod_{i=1}^{n_{1}}\left(x^{2}+\left(m_{2} t+2 t+r_{1} t\right) x+r_{1} m_{2} t^{2}+2 r_{1} t^{2}-\lambda_{i}\left(G_{1}\right)-r_{1}\right) \\
& \cdot \prod_{i=1}^{n_{2}}\left(x^{2}+\left(m_{1} t+2 t+r_{2} t\right) x+r_{2} m_{1} t^{2}+2 r_{2} t^{2}-\lambda_{i}\left(G_{2}\right)-r_{2}\right) \\
& \cdot\left(1-\frac{m_{1} m_{2}\left(x+r_{1} t\right)\left(x+r_{2} t\right)\left(x^{2}+\left(m_{1} t+r_{2} t+2 t\right) x+r_{2} m_{1} t^{2}+2 r_{2} t^{2}-2 r_{2}\right)^{-1}}{\left(x^{2}+\left(m_{2} t+r_{1} t+2 t\right) x+r_{1} m_{2} t^{2}+2 r_{1} t^{2}-2 r_{1}\right)}\right) \\
& \cdot\left(x+m_{1} t+2 t\right)^{m_{2}-n_{2}}\left(x+m_{2} t+2 t\right)^{m_{1}-n_{1}} .
\end{aligned}
$$

Proof. Let $R_{1}$ be the incidence matrix of $G_{1}$. Then, with respect to the adjacent matrix and degree matrix of $G_{1} \odot G_{2}$, the generalized matrix of $G_{1} \odot G_{2}$ is given by

$$
\begin{aligned}
& A_{G_{1} \ominus G_{2}}-t D_{G_{1} \ominus G_{2}} \\
& =\left(\begin{array}{cccc}
-r_{1} t I_{n_{1}} & R_{1} & 0_{n_{1} \times m_{2}} & 0_{n_{1} \times n_{2}} \\
R_{1}^{T} & -\left(m_{2}+2\right) t I_{m_{1}} & \mathbf{1}_{m_{1} \times m_{2}} & 0_{m_{1} \times n_{2}} \\
0_{m_{2} \times n_{1}} & \mathbf{1}_{m_{2} \times m_{1}} & -\left(m_{1}+2\right) t I_{m_{2}} & R_{2}^{T} \\
0_{n_{2} \times n_{1}} & 0_{n_{2} \times m_{1}} & R_{2} & -r_{2} t I_{n_{2}}
\end{array}\right) .
\end{aligned}
$$

Thus the generalized characteristic polynomial of $G_{1} \odot G_{2}$ is

$$
\begin{aligned}
\phi_{G_{1} \odot G_{2}}(x ; t) & =\operatorname{det}\left(\begin{array}{cccc}
\left(x+r_{1} t\right) I_{n_{1}} & -R_{1} & 0_{n_{1} \times m_{2}} & 0_{n_{1} \times n_{2}} \\
-R_{1}^{T} & \left(x+m_{2} t+2 t\right) I_{m_{1}} & -\mathbf{1}_{m_{1} \times m_{2}} & 0_{m_{1} \times n_{2}} \\
0_{m_{2} \times m_{1}} & -\mathbf{1}_{m_{2} \times m_{1}} & \left(x+m_{1} t+2 t\right) I_{m_{2}} & -R_{2}^{T} \\
0_{n_{2} \times n_{1}} & 0_{n_{2} \times m_{1}} & -R_{2} & \left(x+r_{2} t\right) I_{n_{2}}
\end{array}\right) \\
& =\operatorname{det}\left(\left(x+r_{1} t\right) I_{n_{1}}\right) \operatorname{det}(S)=\left(x+r_{1} t\right)^{n_{1}} \operatorname{det}(S),
\end{aligned}
$$

where

$$
S=\left(\begin{array}{ccc}
\left(x+m_{2} t+2 t\right) I_{m_{1}}-\frac{R_{1}^{T} R_{1}}{x+r_{1} t} & -\mathbf{1}_{m_{1} \times m_{2}} & 0_{m_{1} \times n_{2}} \\
-\mathbf{1}_{m_{2} \times m_{1}} & \left(x+m_{1} t+2 t\right) I_{m_{2}} & -R_{2}^{T} \\
0_{n_{2} \times m_{1}} & -R_{2} & \left(x+r_{2} t\right) I_{n_{2}}
\end{array}\right)
$$

is the Schur complement of $\left(x+r_{1} t\right) I_{n_{1}}$. So, $\operatorname{det}(S)=$ $\left(x+r_{2} t\right)^{n_{2}} \operatorname{det}\left(S_{1}\right)$, where

$$
S_{1}=\left(\begin{array}{cc}
\left(x+m_{2} t+2 t\right) I_{m_{1}}-\frac{R_{1}^{T} R_{1}}{x+r_{1} t} & -\mathbf{1}_{m_{1} \times m_{2}} \\
-\mathbf{1}_{m_{2} \times m_{1}} & \left(x+m_{1} t+2 t\right) I_{m_{2}}-\frac{R_{2}^{T} R_{2}}{x+r_{2} t}
\end{array}\right)
$$


is the Schur complement of $\left(x+r_{2} t\right) I_{n_{2}}$. So,

$$
\begin{aligned}
& \operatorname{det}\left(S_{1}\right)=\operatorname{det}\left(\left(x+m_{2} t+2 t\right) I_{m_{1}}-\frac{R_{1}^{T} R_{1}}{x+r_{1} t}\right) \\
& \cdot \operatorname{det}\left(\left(x+m_{1} t+2 t\right) I_{m_{2}}-\frac{R_{2}^{T} R_{2}}{x+r_{2} t}\right. \\
& -\mathbf{1}_{m_{2} \times m_{1}}\left(\left(x+m_{2} t+2 t\right) I_{m_{1}}-\frac{R_{1}^{T} R_{1}}{x+r_{1} t}\right)^{-1} \\
& \left.\cdot \mathbf{1}_{m_{1} \times m_{2}}\right)
\end{aligned}
$$

$$
\begin{aligned}
& \cdot \operatorname{det}\left(\left(x+m_{1} t+2 t\right) I_{m_{2}}-\frac{R_{2}^{T} R_{2}}{x+r_{2} t}\right. \\
& -\mathbf{1}_{m_{2} \times m_{1}}\left(\left(x+m_{2} t+2 t\right) I_{m_{1}}-\frac{R_{1}^{T} R_{1}}{x+r_{1} t}\right)^{-1} \\
& \left.\cdot \mathbf{1}_{m_{1} \times m_{2}}\right)
\end{aligned}
$$

by Lemma 4 ,

$$
\begin{aligned}
& \phi_{G_{1} \ominus G_{2}}(x ; t)=\left(x+r_{1} t\right)^{n_{1}}\left(x+r_{2} t\right)^{n_{2}}\left(x+m_{2} t\right. \\
& +2 t)^{m_{1}-n_{1}} \prod_{i=1}^{n_{1}}\left(\left(x+m_{2} t+2 t\right)\right. \\
& \left.-\frac{\lambda_{i}\left(G_{1}\right)+r_{1}-2+2}{x+r_{1} t}\right) \cdot \operatorname{det}\left(\left(x+m_{1} t+2 t\right) I_{m_{2}}\right. \\
& \left.-\frac{R_{2}^{T} R_{2}}{x+r_{2} t}-\Gamma_{R_{1}^{T} R_{1} /\left(x+r_{1} t\right)}\left(x+m_{2} t+2 t\right) \mathbf{1}_{m_{2} \times m_{2}}\right)
\end{aligned}
$$

and by Lemma 1 ,

$$
\begin{aligned}
\phi_{G_{1} \ominus G_{2}}(x ; t)= & \left(x+r_{1} t\right)^{n_{1}}\left(x+r_{2} t\right)^{n_{2}}\left(x+m_{2} t+2 t\right)^{m_{1}-n_{1}} \prod_{i=1}^{n_{1}}\left(x+m_{2} t+2 t-\frac{\lambda_{i}\left(G_{1}\right)+r_{1}}{x+r_{1} t}\right) \\
& \cdot\left(1-\Gamma_{R_{1}^{T} R_{1} /\left(x+r_{1} t\right)}\left(x+m_{2} t+2 t\right) \Gamma_{R_{2}^{T} R_{2} /\left(x+r_{2} t\right)}\left(x+m_{1} t+2 t\right)\right) \operatorname{det}\left(\left(x+m_{1} t+2 t\right) I_{m_{2}}-\frac{R_{2}^{T} R_{2}}{x+r_{2} t}\right) \\
= & \prod_{i=1}^{n_{1}}\left(x^{2}+\left(m_{2} t+2 t+r_{1} t\right) x+r_{1} m_{2} t^{2}+2 r_{1} t^{2}-\lambda_{i}\left(G_{1}\right)-r_{1}\right) \\
& \cdot \prod_{i=1}^{n_{2}}\left(x^{2}+\left(m_{1} t+2 t+r_{2} t\right) x+r_{2} m_{1} t^{2}+2 r_{2} t^{2}-\lambda_{i}\left(G_{2}\right)-r_{2}\right) \\
& \cdot\left(1-\frac{\left.m_{1} m_{2}\left(x+r_{1} t\right)\left(x+r_{2} t\right)\left(x^{2}+\left(m_{1} t+r_{2} t+2 t\right) x+r_{2} m_{1} t^{2}+2 r_{2} t^{2}-2 r_{2}\right)^{-1}\right)}{\left(x^{2}+\left(m_{2} t+r_{1} t+2 t\right) x+r_{1} m_{2} t^{2}+2 r_{1} t^{2}-2 r_{1}\right)}\right) \\
& \cdot\left(x+m_{1} t+2 t\right)^{m_{2}-n_{2}}\left(x+m_{2} t+2 t\right)^{m_{1}-n_{1}} \cdot
\end{aligned}
$$

Theorem 8. Let $G_{1}$ be an $r_{1}$-regular graph on $n_{1}$ vertices and $m_{1}$ edges and $G_{2}$ an $r_{2}$-regular graph on $n_{2}$ vertices and $m_{2}$ edges. Then

(1) the adjacency characteristic polynomial of $G_{1} \odot G_{2}$ is

$$
\begin{aligned}
\psi & \left(A_{G_{1} \ominus G_{2}} ; x\right)=x^{m_{1}+m_{2}-n_{1}-n_{2}} \prod_{i=1}^{n_{1}}\left(x^{2}-\lambda_{i}\left(G_{1}\right)-r_{1}\right) \\
& \cdot \prod_{i=1}^{n_{2}}\left(x^{2}-\lambda_{i}\left(G_{2}\right)-r_{2}\right) \\
& \cdot\left(1-\frac{m_{1} m_{2} x^{2}}{x^{4}-2\left(r_{1}+r_{2}\right) x^{2}+4 r_{1} r_{2}}\right)
\end{aligned}
$$

(2) the Laplacian characteristic polynomial of $G_{1} \odot G_{2}$ is

$$
\begin{aligned}
& \psi\left(L_{G_{1} \ominus G_{2}} ; x\right)=\prod_{i=2}^{n_{1}}\left(x^{2}-\left(m_{2}+2+r_{1}\right) x+r_{1} m_{2}\right. \\
& \left.+u_{i}\left(G_{1}\right)\right) \prod_{i=2}^{n_{2}}\left(x^{2}-\left(m_{1}+2+r_{2}\right) x+r_{2} m_{1}\right. \\
& \left.+u_{i}\left(G_{2}\right)\right) \cdot\left(x-m_{1}-2\right)^{m_{2}-n_{2}}\left(x-m_{2}-2\right)^{m_{1}-n_{1}} \\
& \quad \cdot x\left(x^{3}-\left(m_{1}+m_{2}+r_{1}+r_{2}+4\right) x^{2}+\left(r_{1} m_{2}\right.\right. \\
& \quad+r_{2} m_{1}+r_{2} m_{2}+r_{1} m_{1}+r_{1} r_{2}+2 m_{1}+2 m_{2}+2 r_{1} \\
& \left.\left.\quad+2 r_{2}+4\right) x-m_{1} r_{1} r_{2}-m_{2} r_{1} r_{2}-2 m_{1} r_{2}-2 m_{2} r_{1}\right),
\end{aligned}
$$


(3) the signless Laplacian characteristic polynomial of $G_{1} \odot G_{2}$ is

$$
\begin{aligned}
\psi\left(Q_{G_{1} \ominus G_{2}} ; x\right)= & \prod_{i=1}^{n_{1}}\left(x^{2}-\left(m_{2}+2+r_{1}\right) x+r_{1}\left(m_{2}+2\right)-v_{i}\left(G_{1}\right)\right)\left(x-m_{2}-2\right)^{m_{1}-n_{1}} \\
& \cdot\left(1-\frac{m_{1} m_{2}\left(x-r_{1}\right)\left(x-r_{2}\right)}{\left(x^{2}-\left(m_{2}+r_{1}+2\right) x+m_{2} r_{1}\right)\left(x^{2}-\left(m_{1}+r_{2}+2\right) x+m_{1} r_{2}\right)}\right) \\
& \cdot \prod_{i=1}^{n_{2}}\left(x^{2}-\left(m_{1}+2+r_{2}\right) x+r_{2}\left(m_{1}+2\right)-v_{i}\left(G_{2}\right)\right)\left(x-m_{1}-2\right)^{m_{2}-n_{2}} .
\end{aligned}
$$

\section{Applications}

We give the number of spanning trees, the Kirchhoff index, and LEL of the two classes of join graphs, as the applications.

We can easily compute the $L$-spectrum of $G_{1} \odot G_{2}$ in terms of $L$-spectrum of $G_{1}$ and $G_{2}$ by (2) of Theorem 6 .

Corollary 9. Let $G_{1}$ be an $r_{1}$-regular graph on $n_{1}$ vertices and $m_{1}$ edges and $G_{2}$ an $r_{2}$-regular graph on $n_{2}$ vertices and $m_{2}$ edges. Then the Laplacian spectrum of $G_{1} \odot G_{2}$ consists of

(1) 2, repeated $m_{1}+m_{2}-n_{1}-n_{2}$ times;

(2) two roots of the equation $x^{2}-\left(r_{1}+n_{2}+2\right) x+2 n_{2}+$ $\mu_{i}\left(G_{1}\right)=0$, for each $i=2, \ldots, n_{1}$;

(3) two roots of the equation $x^{2}-\left(r_{2}+n_{1}+2\right) x+2 n_{1}+$ $\mu_{i}\left(G_{2}\right)=0$, for each $i=2, \ldots, n_{2} ;$

(4) four roots of the equation $x\left(x^{3}-\left(n_{1}+n_{2}+r_{1}+r_{2}+\right.\right.$ 4) $x^{2}+\left(4 n_{1}+4 n_{2}+2 r_{1}+2 r_{2}+4+r_{1} r_{2}+r_{1} n_{1}+r_{2} n_{2}\right) x-$ $\left.4 n_{1}-4 n_{2}-2 n_{1} r_{1}-2 n_{2} r_{2}\right)=0$, and the four roots are $0, \omega_{1}, \omega_{2}$, and $\omega_{3}$, respectively.

We can easily compute the L-spectrum of $G_{1} \odot G_{2}$ in terms of $L$-spectrum of $G_{1}$ and $G_{2}$ by (2) of Theorem 8 .

Corollary 10. Let $G_{1}$ be an $r_{1}$-regular graph on $n_{1}$ vertices and $m_{1}$ edges and $G_{2}$ an $r_{2}$-regular graph on $n_{2}$ vertices and $m_{2}$ edges. Then the Laplacian spectrum of $G_{1} \odot G_{2}$ consists of

(1) $m_{1}+2$, repeated $m_{2}-n_{2}$ times;

(2) $m_{2}+2$, repeated $m_{1}-n_{1}$ times;

(3) two roots of the equation $x^{2}-\left(m_{2}+2+r_{1}\right) x+r_{1} m_{2}+$ $\mu_{i}\left(G_{1}\right)=0$, for each $i=2, \ldots, n_{1}$;

(4) two roots of the equation $x^{2}-\left(m_{1}+2+r_{2}\right) x+r_{2} m_{1}+$ $\mu_{i}\left(G_{2}\right)=0$, for each $i=2, \ldots, n_{2} ;$

(5) four roots of the equation $x\left(x^{3}-\left(m_{1}+m_{2}+r_{1}+r_{2}+\right.\right.$ 4) $x^{2}+\left(r_{1} m_{2}+r_{2} m_{1}+r_{2} m_{2}+r_{1} m_{1}+r_{1} r_{2}+2 m_{1}+2 m_{2}+\right.$ $\left.\left.2 r_{1}+2 r_{2}+4\right) x-m_{1} r_{1} r_{2}-m_{2} r_{1} r_{2}-2 m_{1} r_{2}-2 m_{2} r_{1}\right)=0$, and the four roots are $0, \gamma_{1}, \gamma_{2}$, and $\gamma_{3}$, respectively.

Let $t(G)$ denote the number of spanning trees [1] of $G$. It is well known that if $G$ is a connected graph on $n$ vertices with Laplacian spectrum $0=\mu_{1}(G) \leq \mu_{2}(G) \leq \cdots \leq \mu_{n}(G)$, then $t(G)=\left(\mu_{2}(G) \cdots \mu_{n}(G)\right) / n$.

Corollary 11. Let $G_{1}$ be an $r_{1}$-regular graph on $n_{1}$ vertices and $m_{1}$ edges and $G_{2}$ an $r_{2}$-regular graph on $n_{2}$ vertices and $m_{2}$ edges. The number of spanning trees of $G_{1} \odot G_{2}$ and $G_{1} \odot G_{2}$ are, respectively,

$$
\begin{aligned}
& t\left(G_{1} \odot G_{2}\right)=\frac{2^{m_{1}+m_{2}-n_{1}-n_{2}} \prod_{i=2}^{n_{1}}\left(2 n_{2}+\mu_{i}\left(G_{1}\right)\right) \prod_{i=2}^{n_{2}}\left(2 n_{1}+\mu_{i}\left(G_{2}\right)\right)\left(4 n_{1}+4 n_{2}+2 r_{1} n_{1}+2 r_{2} n_{2}\right)}{n_{1}+m_{1}+n_{2}+m_{2}}, \\
& t\left(G_{1} \odot G_{2}\right)=\frac{\left(m_{1}+2\right)^{m_{2}-n_{2}}\left(m_{2}+2\right)^{m_{1}-n_{1}} \prod_{i=2}^{n_{1}}\left(r_{1} m_{2}+\mu_{i}\left(G_{1}\right)\right) \prod_{i=2}^{n_{2}}\left(r_{2} m_{1}+\mu_{i}\left(G_{2}\right)\right)}{\left(n_{1}+m_{1}+n_{2}+m_{2}\right)\left(m_{1} r_{1} r_{2}+m_{2} r_{1} r_{2}+2 m_{1} r_{2}+2 m_{2} r_{1}\right)^{-1}} .
\end{aligned}
$$

Proof. For $G_{1} \odot G_{2}$, the number of the vertices is $n_{1}+m_{1}+$ $n_{2}+m_{2}$. By Corollary 9 and Vieta's formulas, we can obtain the following: for every $\mu_{i}\left(G_{1}\right), \prod_{i=2}^{n_{1}}\left(x_{1} x_{2}\right)=\prod_{i=2}^{n_{1}}\left(2 n_{2}+\mu_{i}\left(G_{1}\right)\right)$; for every $\mu_{i}\left(G_{2}\right), \prod_{i=2}^{n_{2}}\left(x_{1} x_{2}\right)=\prod_{i=2}^{n_{2}}\left(2 n_{1}+\mu_{i}\left(G_{2}\right)\right)$; for the equation $x^{3}-\left(n_{1}+n_{2}+r_{1}+r_{2}+4\right) x^{2}+\left(4 n_{1}+4 n_{2}+2 r_{1}+\right.$ $\left.2 r_{2}+4+r_{1} r_{2}+r_{1} n_{1}+r_{2} n_{2}\right) x-4 n_{1}-4 n_{2}-2 n_{1} r_{1}-2 n_{2} r_{2}=0$, $\omega_{1} \omega_{2} \omega_{3}=4 n_{1}+4 n_{2}+2 r_{1} n_{1}+2 r_{2} n_{2}$. Therefore, we complete the proof of $t\left(G_{1} \odot G_{2}\right)$ and we omit the proof of $t\left(G_{1} \odot G_{2}\right)$.

The Kirchhoff index [24] $\mathrm{Kf}(G)$ is an important physical and chemical indicator. Let $G$ be a connected graph of order $n$; then $\operatorname{Kf}(G)=n \sum_{i=2}^{n}\left(1 / \mu_{i}\right)$. 
Corollary 12. Let $G_{1}$ be an $r_{1}$-regular graph on $n_{1}$ vertices and $m_{1}$ edges and $G_{2}$ an $r_{2}$-regular graph on $n_{2}$ vertices and $m_{2}$ edges; then the Kirchhoff index of $G_{1} \odot G_{2}$ and $G_{1} \odot G_{2}$ is, respectively,

$$
\begin{aligned}
& \mathrm{Kf}\left(G_{1} \odot G_{2}\right)=\left(m_{1}+n_{1}+m_{2}+n_{2}\right)\left(\frac{m_{1}+m_{2}-n_{1}-n_{2}}{2}+\sum_{i=2}^{n_{1}} \frac{r_{1}+n_{2}+2}{2 n_{2}+\mu_{i}\left(G_{1}\right)}+\sum_{i=2}^{n_{2}} \frac{r_{2}+n_{1}+2}{2 n_{1}+\mu_{i}\left(G_{2}\right)}\right. \\
& \left.\quad+\frac{4 n_{1}+4 n_{2}+2 r_{1}+2 r_{2}+4+r_{1} r_{2}+r_{1} n_{1}+r_{2} n_{2}}{4 n_{1}+4 n_{2}+2 r_{1} n_{1}+2 r_{2} n_{2}}\right), \\
& \mathrm{Kf}\left(G_{1} \odot G_{2}\right)=\left(m_{1}+n_{1}+m_{2}+n_{2}\right)\left(\frac{m_{2}-n_{2}}{2+m_{1}}+\frac{m_{1}-n_{1}}{2+m_{2}}+\sum_{i=2}^{n_{1}} \frac{m_{2}+2+r_{1}}{r_{1} m_{2}+\mu_{i}\left(G_{1}\right)}+\sum_{i=2}^{n_{2}} \frac{m_{1}+2+r_{2}}{r_{2}+\mu_{i}\left(G_{2}\right)}\right. \\
& \left.\quad+\frac{r_{1} m_{2}+r_{2} m_{1}+r_{2} m_{2}+r_{1} m_{1}+r_{1} r_{2}+2 m_{1}+2 m_{2}+2 r_{1}+2 r_{2}+4}{m_{1} r_{1} r_{2}+m_{2} r_{1} r_{2}+2 m_{1} r_{2}+2 m_{2} r_{1}}\right) .
\end{aligned}
$$

Proof. For $G_{1} \odot G_{2}$, by Corollary 9 and Vieta's formulas, we can obtain the following: for every $\mu_{i}\left(G_{1}\right), \sum_{i=2}^{n_{1}}\left(1 / x_{1}+1 / x_{2}\right)=$ $\sum_{i=2}^{n_{1}}\left(\left(x_{1}+x_{2}\right) / x_{1} x_{2}\right)=\sum_{i=2}^{n_{1}}\left(\left(r_{1}+n_{2}+2\right) /\left(2 n_{2}+\mu_{i}\left(G_{1}\right)\right)\right)$; for every $\mu_{i}\left(G_{2}\right), \sum_{i=2}^{n_{2}}\left(1 / x_{1}+1 / x_{2}\right)=\sum_{i=2}^{n_{2}}\left(\left(r_{2}+n_{1}+2\right) /\left(2 n_{1}+\right.\right.$ $\left.\left.\mu_{i}\left(G_{2}\right)\right)\right)$; for the equation $x^{3}-\left(n_{1}+n_{2}+r_{1}+r_{2}+4\right) x^{2}+\left(4 n_{1}+\right.$ $\left.4 n_{2}+2 r_{1}+2 r_{2}+4+r_{1} r_{2}+r_{1} n_{1}+r_{2} n_{2}\right) x-4 n_{1}-4 n_{2}-2 n_{1} r_{1}-2 n_{2} r_{2}=$ $0,1 / \omega_{1}+1 / \omega_{2}+1 / \omega_{3}=\left(\omega_{1} \omega_{2}+\omega_{2} \omega_{3}+\omega_{1} \omega_{3}\right) / \omega_{1} \omega_{2} \omega_{3}=\left(4 n_{1}+\right.$ $\left.4 n_{2}+2 r_{1}+2 r_{2}+4+r_{1} r_{2}+r_{1} n_{1}+r_{2} n_{2}\right) /\left(4 n_{1}+4 n_{2}+2 r_{1} n_{1}+2 r_{2} n_{2}\right)$. Therefore, we complete the proof of $\operatorname{Kf}\left(G_{1} \odot G_{2}\right)$ and we omit the proof of $\mathrm{Kf}\left(G_{1} \odot G_{2}\right)$.

$\operatorname{LEL}(G)$ denotes Laplacian-Energy-Like Invariant [24]. Let $G$ be a connected graph of order $n$ with $m$ edges; then $\operatorname{LEL}=\operatorname{LEL}(G)=\sum_{i=2}^{n} \sqrt{\mu_{i}}$.

Corollary 13. Let $G_{1}$ be an $r_{1}$-regular graph on $n_{1}$ vertices and $m_{1}$ edges and $G_{2}$ an $r_{2}$-regular graph on $n_{2}$ vertices and $m_{2}$ edges; then

$$
\begin{aligned}
\operatorname{LEL}\left(G_{1} \odot G_{2}\right)= & \sqrt{2}\left(m_{1}+m_{2}-n_{1}-n_{2}\right)+\sum_{i=1}^{3} \sqrt{\omega_{i}} \\
& +\sum_{i=2}^{n_{1}} \sqrt{\frac{r_{1}+n_{2}+2 \pm \sqrt{\Delta_{1}}}{2}} \\
& +\sum_{i=2}^{n_{2}} \sqrt{\frac{r_{2}+n_{1}+2 \pm \sqrt{\Delta_{2}}}{2}}
\end{aligned}
$$

where $\Delta_{1}=\left(r_{1}+n_{2}+2\right)^{2}-4\left(2 n_{2}+\mu_{i}\left(G_{1}\right)\right), \Delta_{2}=\left(r_{2}+n_{1}+\right.$ $2)^{2}-4\left(2 n_{1}+\mu_{i}\left(G_{2}\right)\right)$, and $\omega_{1}, \omega_{2}$, and $\omega_{3}$ are three roots of the equation $x^{3}-\left(n_{1}+n_{2}+r_{1}+r_{2}+4\right) x^{2}+\left(4 n_{1}+4 n_{2}+2 r_{1}+\right.$ $\left.2 r_{2}+4+r_{1} r_{2}+r_{1} n_{1}+r_{2} n_{2}\right) x-4 n_{1}-4 n_{2}-2 n_{1} r_{1}-2 n_{2} r_{2}=$ 0 .

$$
\begin{aligned}
\operatorname{LEL}\left(G_{1} \odot G_{2}\right)= & \left(m_{2}-n_{2}\right) \sqrt{m_{1}+2} \\
& +\left(m_{1}-n_{1}\right) \sqrt{m_{2}+2}+\sum_{i=1}^{3} \sqrt{\gamma_{i}}
\end{aligned}
$$

$$
\begin{aligned}
& +\sum_{i=2}^{n_{1}} \sqrt{\frac{m_{2}+2+r_{1} \pm \sqrt{\Delta_{3}}}{2}} \\
& +\sum_{i=2}^{n_{2}} \sqrt{\frac{m_{1}+2+r_{2} \pm \sqrt{\Delta_{4}}}{2}},
\end{aligned}
$$

where $\Delta_{3}=\left(m_{2}+2+r_{1}\right)^{2}-4\left(r_{1} m_{2}+\mu_{i}\left(G_{1}\right)\right), \Delta_{4}=\left(m_{1}+\right.$ $\left.2+r_{2}\right)^{2}-4\left(r_{2} m_{1}+\mu_{i}\left(G_{2}\right)\right)$, and $\gamma_{1}, \gamma_{2}$, and $\gamma_{3}$ are three roots of the equation $x^{3}-\left(m_{1}+m_{2}+r_{1}+r_{2}+4\right) x^{2}+\left(r_{1} m_{2}+r_{2} m_{1}+\right.$ $\left.r_{2} m_{2}+r_{1} m_{1}+r_{1} r_{2}+2 m_{1}+2 m_{2}+2 r_{1}+2 r_{2}+4\right) x-m_{1} r_{1} r_{2}-$ $m_{2} r_{1} r_{2}-2 m_{1} r_{2}-2 m_{2} r_{1}=0$.

\section{Conclusions}

In this paper, two classes of join graphs, $G_{1} \odot G_{2}$ and $G_{1} \odot G_{2}$, are constructed by graph operations. The formulas of generalized characteristic polynomial of the two graphs are obtained by using block matrix and Schur complement. For regular graphs $G_{1}$ and $G_{2}$, we characterize the $A-, L$ , and $Q$-spectrum of $G_{1} \odot G_{2}$ and $G_{1} \odot G_{2}$ in terms of the corresponding spectrum of $G_{1}$ and $G_{2}$, and by the $L$ spectrum, we get the number of spanning trees, Kirchhoff index, and LEL. But for nonregular graphs $G_{1}$ and $G_{2}$, we cannot determine the spectrum of $G_{1} \odot G_{2}$ and $G_{1} \odot G_{2}$. The most difficult problem is that we cannot find the rule of the block matrices to deduce a simple result. Therefore, we should try to find new method to solve the problem.

\section{Competing Interests}

The authors declare that they have no competing interests.

\section{Acknowledgments}

This paper is supported by the National Natural Science Foundation of China (no. 11361033). 


\section{References}

[1] D. S. Cvetkovi, M. Doob, and H. Sachs, Spectra of Graphs. Theory and Applications, Johann Ambrosius Barth, Heidelberg, Germany, 3rd edition, 1995.

[2] D. J. Klein and M. Randić, "Resistance distance," Journal of Mathematical Chemistry, vol. 12, no. 1, pp. 81-95, 1993.

[3] I. Gutman and B. Mohar, "The quasi-Wiener and the Kirchhoff indices coincide," Journal of Chemical Information and Computer Sciences, vol. 36, no. 5, pp. 982-985, 1996.

[4] R. B. Bapat and S. Gupta, "Resistance distance in wheels and fans," Indian Journal of Pure and Applied Mathematics, vol. 41, no. 1, pp. 1-13, 2010.

[5] J.-B. Liu, X.-F. Pan, and F.-T. Hu, "The 1-inverse of the Laplacian of subdivision-vertex and subdivision-edge coronae with applications," Linear and Multilinear Algebra, vol. 65, no. 1, pp. 178-191, 2017.

[6] Y. Yang, "The Kirchhoff index of subdivisions of graphs," Discrete Applied Mathematics. The Journal of Combinatorial Algorithms, Informatics and Computational Sciences, vol. 171, pp. 153-157, 2014.

[7] Y. Yang and D. J. Klein, "A recursion formula for resistance distances and its applications," Discrete Applied Mathematics, vol. 161, no. 16-17, pp. 2702-2715, 2013.

[8] Y. Yang and D. J. Klein, "Resistance distance-based graph invariants of subdivisions and triangulations of graphs," Discrete Applied Mathematics, vol. 181, pp. 260-274, 2015.

[9] J. Liu and B. Liu, "A Laplacian-energy-like invariant of a graph," MATCH. Communications in Mathematical and in Computer Chemistry, vol. 59, no. 2, pp. 355-372, 2008.

[10] I. Gutman, B. Zhou, and B. Furtula, "The Laplacian-energy like invariant is an energy like invariant," MATCH. Communications in Mathematical and in Computer Chemistry, vol. 64, no. 1, pp. 85-96, 2010.

[11] B. Arsi, I. Gutman, K. C. Das, and K. Xu, "Relations between Kirchhoff index and Laplacian-energ-like invariant," Bulletin de l'Académie Serbe des Sciences et des Arts, Classe des Sciences Médicales, vol. 144, pp. 61-72, 2012.

[12] K. C. Das, K. Xu, and I. Gutman, "Comparison between Kirchhoff index and the Laplacian-energy-like invariant," Linear Algebra and Its Applications, vol. 436, no. 9, pp. 3661-3671, 2012.

[13] S.-Y. Cui and G.-X. Tian, "The spectrum and the signless Laplacian spectrum of coronae," Linear Algebra and Its Applications, vol. 437, no. 7, pp. 1692-1703, 2012.

[14] X. G. Liu, J. Zhou, and C. J. Bu, "Resistance distance and Kirchhoff index of R-vertex join and R-edge join of two graphs," Discrete Applied Mathematics, vol. 187, pp. 130-139, 2015.

[15] X. Liu and S. Zhou, "Spectra of the neighbourhood corona of two graphs," Linear and Multilinear Algebra, vol. 62, no. 9, pp. 1205-1219, 2014.

[16] C. McLeman and E. McNicholas, "Spectra of coronae," Linear Algebra and its Applications, vol. 435, no. 5, pp. 998-1007, 2011.

[17] S. Wang and B. Zhou, "The signless Laplacian spectra of the corona and edge corona of two graphs," Linear and Multilinear Algebra, vol. 61, no. 2, pp. 197-204, 2013.

[18] G. Wilkinson, M. Rosenblum, M. C. Whiting, and R. B. Woodward, "The structure of iron bis-cyclopentadienyl," Journal of the American Chemical Society, vol. 74, no. 8, pp. 2125-2126, 1952.

[19] D. M. Cvetkovi, P. Rowlinson, and H. Simi, An Introduction to the Theory of Graph Spectra, Cambridge University Press, Cambridge, UK, 2010.
[20] X. Liu and P. Lu, "Spectra of subdivision-vertex and subdivisionedge neighbourhood coronae," Linear Algebra and Its Applications, vol. 438, no. 8, pp. 3547-3559, 2013.

[21] X. G. Liu and Z. H. Zhang, "Spectra of subdivision-vertex and subdivision-edge joins of graphs," https://arxiv.org/abs/ $1212.0619 \mathrm{v} 3$.

[22] F. Z. Zhang, The Schur Complement and Its Applications, Numerical Methods and Algorithms, Springer-Verlag, New York, NY, USA, 2005.

[23] R. A. Horn and C. R. Johnson, Topics in Matrix Analysis, Cambridge University Press, 1991.

[24] S. Pirzada, H. A. Ganie, and I. Gutman, "On Laplacian energy like invariant and Krichhoff index," MATCH Communications in Mathematical and in Computer Chemistry, vol. 73, pp. 41-59, 2015. 


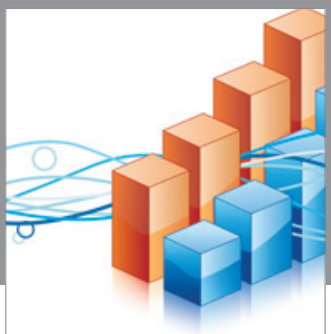

Advances in

Operations Research

vatem alat4

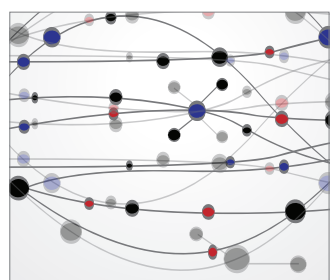

\section{The Scientific} World Journal
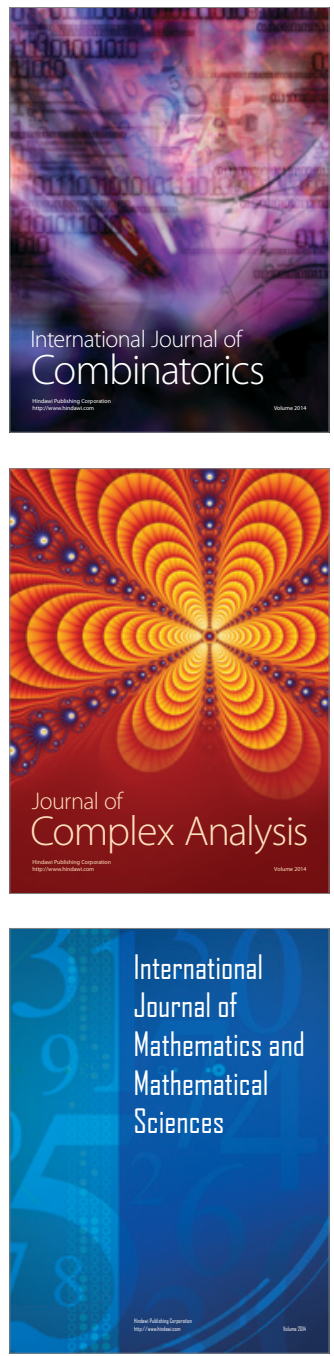
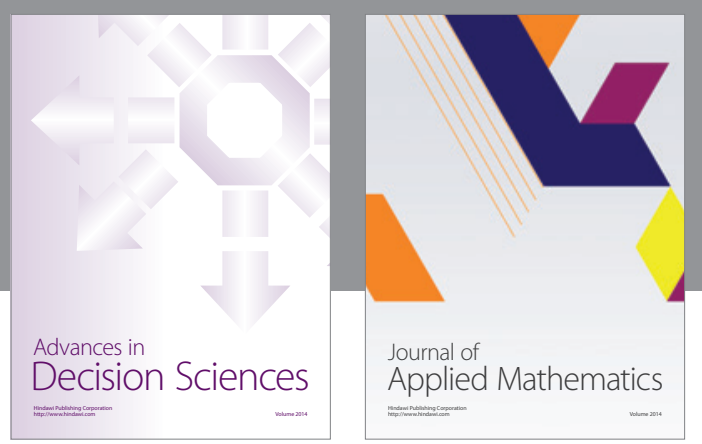

Algebra

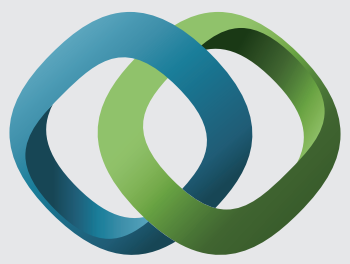

\section{Hindawi}

Submit your manuscripts at

https://www.hindawi.com
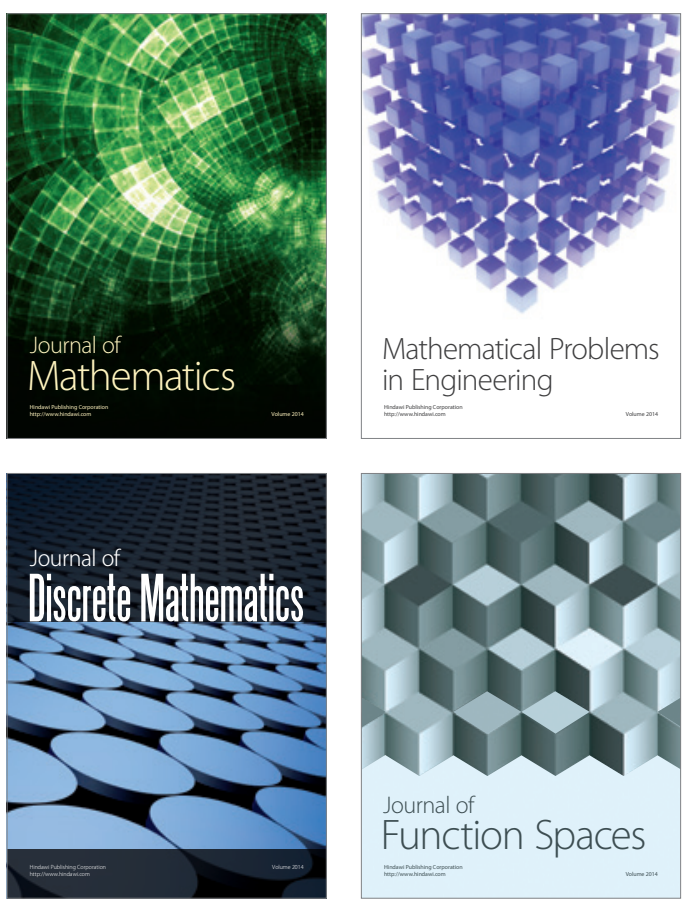

Mathematical Problems in Engineering
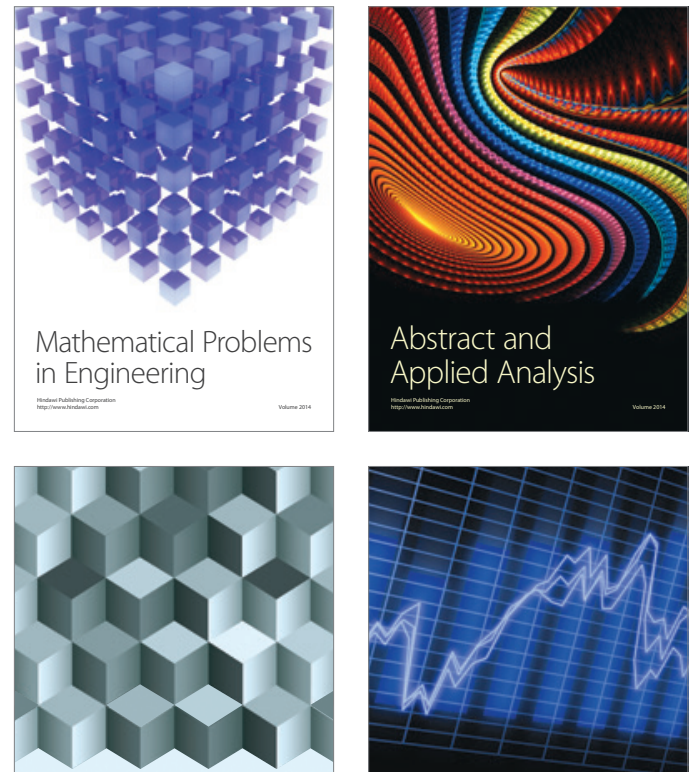

Journal of

Function Spaces

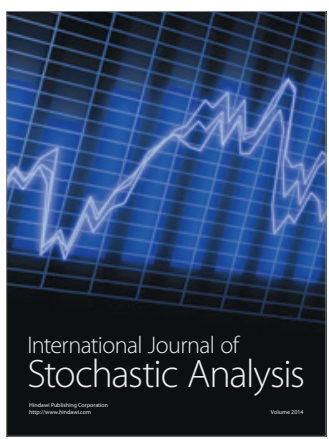

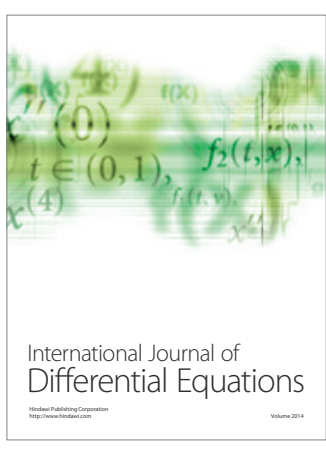
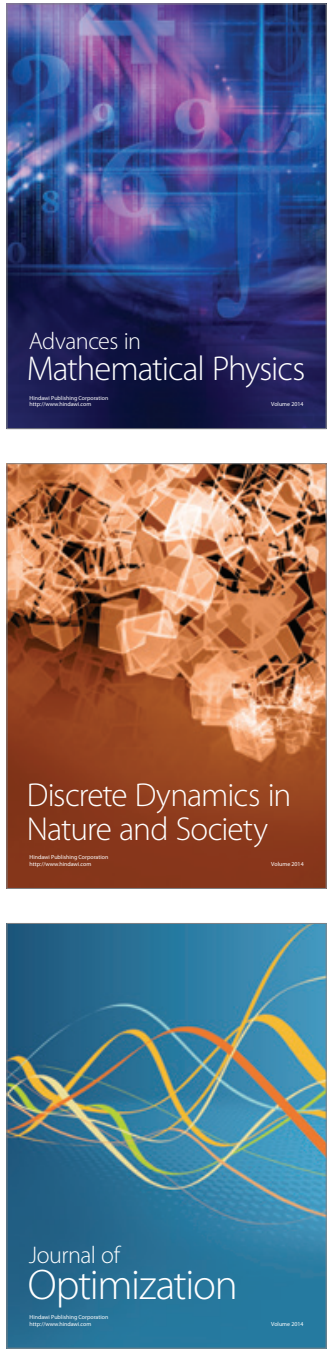